\title{
Bergsoniana
}

1 | 2021

Reassessing Bergson

\section{Bergson and the Heptapods}

\section{Barry Dainton}

\section{OpenEdition}

\author{
Journals
}

\section{Electronic version}

URL: https://journals.openedition.org/bergsoniana/473

DOI: 10.4000/bergsoniana.473

ISSN: 2800-874X

\section{Publisher}

Société des amis de Bergson

\section{Electronic reference}

Barry Dainton, "Bergson and the Heptapods", Bergsoniana [Online], 1 | 2021, Online since 01 July 2021 connection on 08 November 2021. URL: http://journals.openedition.org/bergsoniana/473 ; DOI: https://doi.org/10.4000/bergsoniana.473

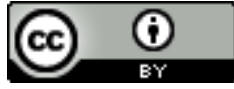

Les contenus de la revue sont mis à disposition selon les termes de la Licence Creative Commons Attribution 4.0 International. 


\title{
BERGSON AND THE HEPTAPODS
}

\author{
Barry DAINTON
}

In their provocative "We Bergsonians: the Kyoto Manifesto," During and Miquel propose the following:

We are setting out the case for an expanded Bergsonism. "Expanded" in what sense? First and foremost, our Bergsonism must engage fully with the sciences of today. It is important to remember that Bergson himself was continuously stimulated and spurred on by the sciences of his time. His method risks amounting to no more than idle play of concepts if it is confined to purely intra-philosophical issues [...] There is an immediate advantage to adopting a broader, expanded Bergsonism: those who find much to admire in Bergson, but who are unable to count themselves as his disciples, need no longer suffer from a guilty conscience. To extend one of his intuitions in a different direction, or find new avenues of attack, nothing forbids us from reconstructing his doctrines, or finding novel ways of recombining his concepts (During et al. 2020, 18-19).

What follows is an attempt to implement this manifesto commitment. The conclusion I will be working towards is that the recently published Beyond the Dynamical Universe, by Silberstein, Stuckey and McDevitt (2018) may well constitute a noteworthy example of the expanded or enlarged Bergsonism that During and Miquel are asking for. Making this claim intelligible, let alone plausible, will take a little preparatory work.

\section{Physics for Heptapods}

The recent movie Arrival (Villeneuve 2016) is interesting for a number of reasons. In the first instance it is a "First Contact" story: a race of hitherto unknown aliens pay us a visit. Overnight twelve huge spaceships approach the Earth and land at (or rather hover over) different locations on the 
Earth's surface. The aliens in question turn out to be really alien: large lumbering seven-limbed creatures - hence the name given to them by the US contingent: "heptapods." The movie focuses on linguist Louise Banks, who is drafted in to help master the aliens' language. Banks, assisted by physicist Ian Donnelly, succeeds in mastering both the spoken and written forms of the alien's language - but only after overcoming numerous hurdles. She subsequently enters into a relationship with Donnelly, and a few years later the couple have a daughter, Hannah.

Louise's efforts are initially hindered by her failure to appreciate that the aliens use totally different languages for speech and writing - a sentence of the latter is shown in Figure 1. The aliens also relate to time in a very different way than we humans are accustomed to doing: they are able to see into the future in something like the way we are able to recall past events. Since Louise starts to acquire this ability as she becomes more fluent in the alien's languages we have an intriguing illustration — albeit fictional — of the Sapir-Whorf thesis that language can determine modes of cognition, especially when it pertains to space and time.

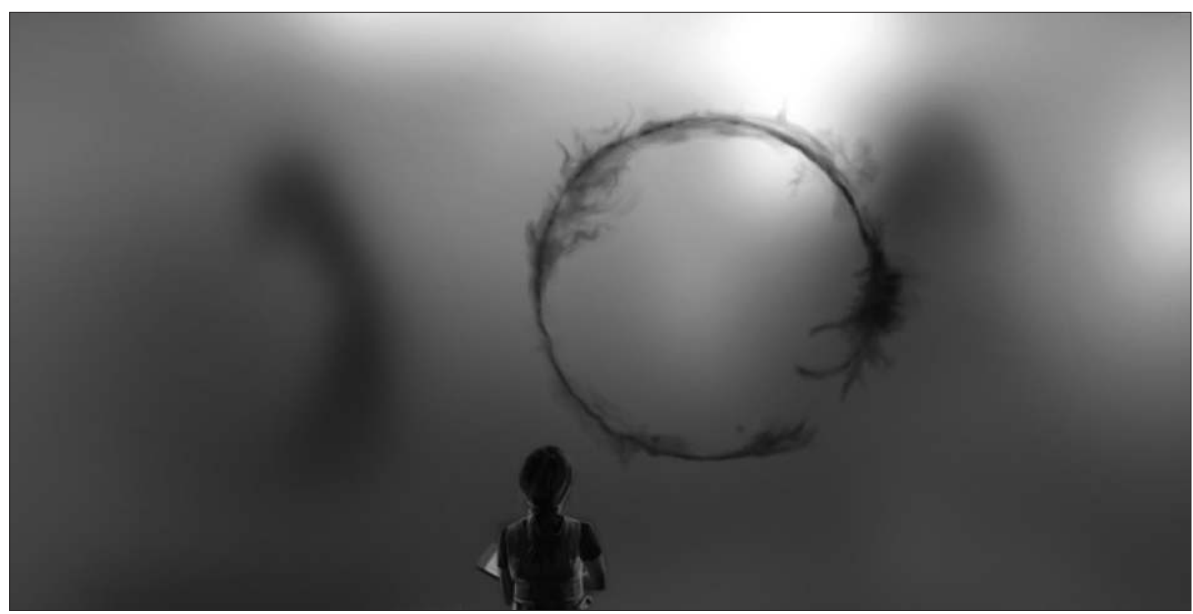

FIGURE 1. A sample of Heptapod writing Image reproduced with the permission of Sony

The plot of Arrival is based on a science fiction novella: Ted Chiang's "Story of My Life." In the latter Chiang provides a more detailed account of the peculiarities of the aliens' spoken and written languages. For present purposes, however, it's a pity that the movie downplays - almost to the 
point of non-existence - another important strand of Chiang's own version of the tale: the heptapod's distinctive physics. In "Story of My Life" Chiang gives physicist Donnelly a larger role to play than he enjoys in the movie. Given that the aliens have solved the problem of interstellar travel, the investigating team are understandably very interested in their science, which is obviously considerably more advanced than our own. After enough of the heptapod language has been mastered to permit rudimentary communication Donnelly eventually learns that the aliens find some parts of our physics very easy to understand, while finding other parts totally baffling — and he also works out why this is so.

Why do material things behave as they do? In addressing this question we are used to thinking in terms of cause and effect. An apple falls when dropped because it being attracted to the centre of the Earth by gravitational force; another object moves when struck or pulled. The (highly successful) mechanical laws formulated by Newton all concern how the behaviour of material objects can be changed under the influence of forces - where the impact of the forces unfolds from one moment to the next moment.

However there is a very different - but equally successful - way of explaining why things behave as they do: instead of local rules we rely instead on global constraints and so-called "variational principles." Fermat announced the first of these in 1662. According to Fermat's Principle when a ray of light moves between two locations in space it follows the path which will take least time. Maupertuis and Euler took the next steps, proposing that nature was governed by principle of "least action," which Maupertuis characterised thus:

[T] he action is neither the speed nor the distance taken separately; rather it is proportional to the sum of the distances travelled each multiplied by the speed at which they were travelled. Hence action increases linearly with speed and with the distance travelled. The action is the true expense of nature, which she manages to make as small as possible (Maupertuis 1744).

This action-minimising approach to classical mechanics was refined and generalised in Lagrange's 1788-89 Mécanique Analytique, where it forms the basis for an alternative formulation of Newton's mechanics. We find there the modern notion that an object's action $S$ is the kinetic energy minus potential energy integrated over time. For each path there is a different value for the total action - the actual path taken is the one with the most minimal action. As Feynman puts it in his lectures on physics: "the laws of Newton could be stated not in the form $F=$ ma but in the 
form: the average kinetic energy less the average potential energy is as little as possible for the path of an object going from one point to another" (Feynman 2011, ch. 19).

\section{From Physics to Metaphysics}

On Earth we have discovered both the local rules and global-constraint ways of doing physics - and in fact discovered the local version before the global version. The heptapods in "Story of Your life," it turns out, never discovered the local approach to physics, they only have the global - and find the local approach almost impossible to grasp. As Chiang relates the tale, Louise finds herself wondering how the heptapods might experience the world, given that they find the least action principles so entirely natural. Here she is conversing with Donnelly:

"Though I did want to ask you about Fermat's Principle. Something about it feels odd to me, but I can't put my finger on it. It just doesn't sound like a law of physics."

A twinkle appeared in Gary's eyes. "I'll bet I know what you're talking about." He snipped a potsticker in half with his chopsticks. "You're used to thinking of refraction in terms of cause and effect: reaching the water's surface is the cause, and the change in direction is the effect. But Fermat's Principle sounds weird because it describes light's behavior in goal-oriented terms. It sounds like a commandment to a light beam: 'Thou shalt minimize or maximize the time taken to reach thy destination."”

I considered it. "Go on."

"It's an old question in the philosophy of physics. People have been talking about it since Fermat first formulated it in the 1600s; Planck wrote volumes about it. The thing is, while the common formulation of physical laws is causal, a variational principle like Fermat's is purposive, almost teleological."

"Hmm, that's an interesting way to put it. Let me think about that for a minute." I pulled out a felt-tip pen and, on my paper napkin, drew a copy of the diagram that Gary had drawn on my blackboard. "Okay," I said, thinking aloud, "so let's say the goal of a ray of light is to take the fastest path. How does the light go about doing that?"

"Well, if I can speak anthropomorphic-projectionally, the light has to examine the possible paths and compute how long each one would take." He plucked the last potsticker from the serving dish.

"And to do that," I continued, "the ray of light has to know just where its destination is. If the destination were somewhere else, the fastest path would be different."

Gary nodded again. "That's right; the notion of a 'fastest path' is meaningless unless there's a destination specified. And computing how long a given path takes 
also requires information about what lies along that path, like where the water's surface is."

I kept staring at the diagram on the napkin. "And the light ray has to know all that ahead of time, before it starts moving, right?"

"So to speak," said Gary. "The light can't start traveling in any old direction and make course corrections later on, because the path resulting from such behavior wouldn't be the fastest possible one. The light has to do all its computations at the very beginning."

I thought to myself, "The ray of light has to know where it will ultimately end up before it can choose the direction to begin moving in. I knew what that reminded me of." I looked up at Gary. "That's what was bugging me" (Chiang 2002, 155-156).

In Arrival — and Chiang's short story - the metaphysical implications of all this are clearly and vividly spelt out: since the Heptapods can perceive the future as well as the past, the future is just as real as the past (or present). Donnelly sometimes talks in terms of light rays "knowing" where they will ultimately end up and "choosing" their ultimate destinations. This way of talking is natural enough when describing the workings of variational principles, but needn't be taken literally. It's perfectly possible to take (for example) the principle of least action as simply an accurate way of describing the behaviour of material objects — such as elementary particles - that are devoid of any trace of mentality or volition. When viewed in this manner there is equally no need to invoke any mysterious "final causes" emanating from a future destination backwards in time. Indeed, if we do think of an object being directed or controlled by its future state we are guilty of illegitimately extending the local cause and effect way of understanding physical law. To ask "How does a moving particle know which is the action-minimizing path given that its ultimate destination lies in the future?" is misguided. Variational principles shouldn't be viewed as operating at the level of local causes at all. They are better viewed simply as regularities or patterns that characterise an atemporal four-dimensional reality.

Since the heptapods are merely creatures of a science fiction story, this has no implications for how we should conceive of the real world. That said, variational principles truly are widely applicable in the real world. Does this fact have any metaphysical implications for how we should conceive of our reality?

One thing, I think, is clear. If the real world were as the heptapods believe it to be, and only governed by least action principles, then we would have no option but to adopt the four-dimensional view of the universe, and 
hold that the future is just as real as the past or present. As Gary Donnelly nicely puts it in the quote given above when he tells us that "the notion of a 'fastest path' is meaningless unless there's a destination specified." Variational principles all minimise or maximise some quantity — whether it's action, energy or time - and the quantity in question is a property of an object's possible trajectories between a specific starting location and a specific destination. Since the end-points of these trajectories or paths exist at earlier and later times it is difficult to see how to make any sense of this unless we admit that both are fully real.

\section{The Grammar of the Universe}

Physicists in our world are faced with a choice of theoretical frameworks that is not available to their heptapod counterparts: we have both the classical Newtonian (local) approach and the (global) least action approach, and both work equally well - the two are provably mathematically equivalent. Consequently, a typical sequence of physical events can be described equally well in the two different ways. This is how Chiang characterises our predicament:

The physical universe was a language with a perfectly ambiguous grammar. Every physical event was an utterance that could be parsed in two entirely different ways, one causal and the other teleological, both valid, neither one disqualifiable no matter how much context was available. When the ancestors of humans and heptapods first acquired the spark of consciousness, they both perceived the same physical world, but they parsed their perceptions differently; the worldviews that ultimately across were the end result of that divergence. Humans had developed a sequential mode of awareness, while heptapods had developed a simultaneous mode of awareness. We experienced events in an order, and perceived their relationship as cause and effect. They experienced all events at once, and perceived a purpose underlying them all. A minimising, maximising purpose (Chiang 2002, 165-166).

If grammar of the language spoken by the physical universe truly were perfectly ambiguous it would be illegitimate to conclude that the universe is four-dimensional simply because physical processes conform to variational principles. If the classical Newtonian view that objects move as they do because of the influence of moment-to-moment forces impinging on them is just as valid as the variational alternative we would not be in a position to claim that the latter provides the truer insight into the temporal character of the universe. 
However, we no longer live in the classical world of Newton. Our current best theory of matter is known as the Standard Model of Particle Physics, which takes the form of a quantum field theory (QFT). This field theory originated in work done on quantisation of the electromagnetic field in 1926-7 by Born, Heisenberg, Jordan and Dirac, and was gradually extended to cover other forces and fields over the next half century or so. The Standard Model received a noteworthy — and much publicised - confirmation when the long-predicted Higgs boson was discovered at CERN in 2013.

According to the Standard Model all material things are composed of three families of particles: quarks, leptons (e.g., electrons and neutrinos) and force carrying bosons (such as electrons and muons). Hadrons are particles made up of multiple quarks: the baryons have three quark constituents - e.g., the protons and neutrons familiar from chemistry fall into this category, whereas the generally short-lived mesons - such as the pion - are composed of just two quarks. QFTs are so-called because their fundamental ingredients are entities known as quantum fields, and particles tend to be viewed as nothing more than patterns of activity within these fields — with different species of particle being associated with different types of quantum field. From the perspective of QFT the universe consists of a number of different overlapping quantum fields each of which extends through all of space. The Standard Model provides an account of three of the known four forces in nature. These are the strong force which binds the quarks, the weak force responsible for the transformation of massive quarks and leptons to lighter particles, and the more familiar electromagnetic force, which has a potentially infinite range.

Why is any of this of relevance to our current concerns? Simply because the standard forms of quantum field theory are entirely based on variational principles. Here's a schematic way of representing the physical principles at the heart of the standard model:

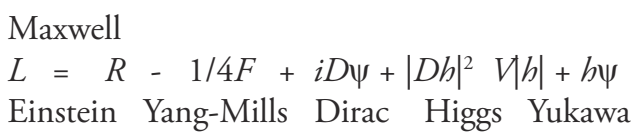

There is much that is new and unfamiliar here: the "Einstein" term describes gravity; "Maxwell" describes electromagnetic forces, and a generalised form of it in the form of the "Yang-Mills" term describes the strong and weak nuclear forces; "Dirac" describes the fermions (e.g., electrons, quarks, neutrinos) — and the "Higgs" and "Yukawa" terms provide these particles 
with mass. But there is also something very familiar from classical physics: the " $L$ " means we're dealing with a "Lagrangian" and hence an actionminimising variational principle.

On opening any textbook on the quantum field theory you will find chapter after chapter are devoted to "Lagrangeans" and "Hamiltonians." The debates among theorists are not about whether or not to apply variational principles or a classical local alternative, they're about which variational principle to apply.

If the language in which nature is written is quantum field theory, and the latter is essentially variational in character, then the lesson is obvious: the language in which nature is written presupposes that our reality is fundamentally atemporal. We should accept that the future is as real as the past or present.

There is an important caveat here. We know that the Standard Model is by no means the last word in physics. Quite what the best mathematical formulation of it will turn out to be remains controversial - there are a number of competing alternatives. Calculations using the theory tend to produce physically unrealistic infinities; although these have been partially tamed by "perturbation" techniques the suspicion remains that a better theory will not have this consequence. The Standard Model includes a large number of parameters that need to be determined experimentally - the theory provides no clue as to why these parameters have these particular values rather than others. Also, and perhaps most importantly, the problem of reconciling quantum theory with general relativity remains unsolved, despite receiving the attentions of many of the best minds in physics over a period of many years.

It's true that since no one yet knows what a viable quantum gravity theory will look like we are similarly ignorant as to the character of the successful theory which will succeed the Standard Model. However, given that variational principles underlie both general relativity and quantum field theory, it does not seem unreasonable to suppose they will also play an important role in the physics of the future — in which case their ontological implications for time remain unchanged. ${ }^{1}$

1. The problems confronting quantum field theories in the current form are such that some theorists are seeking more radical alternatives. Since these alternatives often take the form of attempts to demonstrate that space and time (as we normally conceive them) are merely emergent phenomena, deriving from a fundamental reality which is itself neither spatial nor temporal. It goes without saying that theoretical speculations along 


\section{An Adynamical Blockworld}

In their recent Beyond the Dynamical Universe, Silverstein, Stuckey and McDevitt ("SSM" hereafter) offer what they claim is a "new paradigm" for both physics and metaphysics. At the centre of their metaphysics is something called the Blockworld, conceived in a particular way:

We are not suggesting that general relativity (GR) or quantum field theory (QFT) are wrong, but that they are merely incomplete. The new paradigm we propose is adynamical explanation in a block universe and we offer a specific example called Relational Blockworld (RBW). In terms of the nature of reality itself, we are proposing a wholesale replacement of the mechanical universe (as fundamental) with the ontology of RBW wherein not time-evolved entities, but 4-dimensional spacetime entities are fundamental (Silberstein et al. 2018, 3).

The " $\mathrm{B}$ " in RBW is familiar enough: it is simply the universe viewed in the four-dimensional eternalist manner. The " $R$ " or relational component refers to their rejection of a background spatio-temporal framework that is independent of material objects or events. Following in the footsteps of Leibniz rather than Newton they hold that facts about space and time are grounded in facts about relationships between objects and events. An important aspect of SSM's position is their favouring "adynamical explanation." As for quite what this involves, there are three main elements. First, SSM endorse a robustly Humean conception of causation. Irrespective of how we tend to think, in reality there is no causal "oomph," nothing ever makes anything else happen - there are merely constant conjunctions and regular successions. ${ }^{2}$ Second, they hold that dynamic explanations are not fundamental. We explain something dynamically when we explain the present solely in terms of the past. The Newtonian paradigm is manifestly dynamic in this sense: at any given instant the universe consists of a collection of objects interacting with one another, and the laws of nature specify how such systems evolve from one moment to the next. The adynamical mode of explanation preferred by SSM relies

these lines pose at least as much threat to our ordinary ways of thinking about time as any other. In this connection it is worth noting the recent (and remarkable) successes of the geometrical approach to calculations in quantum field theory by Nima Arkami-Hamed — see Wolchover (2013) for futher details.

2. They also approvingly cite Le Poidevin when he remarks "If the future is real [...] we can no longer view causation as a matter of making something real. We must settle for a watered-down conception of causation" (Le Poidevin 2013, 545). 
on Lagrangean-style variational principles we encountered in the previous section - the sort that lie at the basis of heptapod physics. ${ }^{3}$

Thirdly, the RBW approach rejects the principle that causes only act locally. SSM see nothing illegitimate or undesirable about action-at-a-distance or "direct action" as they call it. Why think the global variational principles responsible for the patterning among events in our four-dimensional universe only permit correlations between spatially adjacent objects and events? When we learn to appreciate that correlations between spatially distant events never involve the instantaneous transmission of causal influence - which simply does not exist in the adynamical blockworld - it is difficult to see why direct action should be thought to be more problematic metaphysically than local action.

Having set out this framework, in the rest of their book SSM go on argue that moving from the dynamical to the adynamical mode of explanation helps resolve some of the most baffling problems facing contemporary physics. In their discussion of quantum theory they argue that otherwise baffling phenomena such as Bell-type entanglement turn out to be quite unproblematic when viewed from the adynamical perspective. While I am not competent to judge whether the approach SSM adopt to quantum gravity, dark matter and dark energy really constitutes a breakthrough, what they say about time and temporal experience does strike me as a significant advance - at least when compared with other recent physicsoriented treatments of these topics.

SSM fully appreciate that the character of our ordinary experience constitutes a potentially serious challenge to their blockworld view. From a phenomenological perspective, our experience is highly dynamic: we can see birds flying through the sky, people walking across the street, musical notes succeed one another - and so on and on. Different forms of motion and succession are ubiquitous features of our experience. How is this reconcilable with the passage-free adynamical blockworld?

Previous defences of blockworld views have tended to appeal to two forms of reductionism - either singly or in combination. If consciousness can be reduced to something wholly lacking in phenomenal or experiential

3. As SSM put it, on this approach the "formalism that is most consistent with adynamical explanation and the block universe ontology is one in which the final state is an input on equal footing with the initial state, and there is some mathematical formulation for the global rule for the spatiotemporal pattern connecting these initial and final states" (SSM 2018 , 9). Wharton (2015) advocates adopting a similar approach. 
features, e.g., to neural or computational processes, then the dynamic temporal features of consciousness would no longer be problematic integrating neural or computational processes into a blockworld is quite straightforward. An alternative approach is to explain away the potentially troublesome appearances of dynamism in our ordinary experiences. This sort of reductionist will hold, for example, that our so-called perceptions of motion or change consist of series of entirely static experiential snapshots - of a sort that is fully compatible with the blockworld view.

SSM reject both forms of reductionism, opting instead for a fullyfledged realism and non-reductionism concerning both the existence of consciousness, and the inherently dynamic and flowing character of experience. Given the lack of success of the various attempts over the course of the $20^{\text {th }}$ century to reduce consciousness to something else, SSM maintain that that the most plausible option is to accept that phenomenal properties are themselves physical. If they are not amongst the properties of elementary particles and fields listed in physics or chemistry textbooks it is because the basic constituent of matter — or at least some of them - have properties over and above those commonly recognised by the physical sciences. In adopting this stance SSM are influenced by much the same considerations which have led contemporary philosophers such as Strawson and Seager to embrace forms of panpsychism - this they acknowledge, but prefer the term "neutral monism."

\section{A Framework for an Expanded Bergsonism?}

I won't go any further into the details of the views SSM develop in Beyond the Dynamical Universe. The question I want to consider by way of a conclusion is whether the general position they defend - i.e., the particular combination of stances they endorse - is one that anyone interested in developing an enlarged or expanded Bergsonism should take seriously.

No doubt some will find this idea absurd. SSM's blockworld is a universe where the future is fully real; from an ontological perspective future events in a blockworld are as set in stone as any past event. ${ }^{4}$ Since the doctrine that the future is open and yet-to-be determined is one of the central tenets of orthodox Bergsonism, how can the latter possibly be reconcilable with

4. SSM certainly make no attempt to conceal the radical consequences of their stance: "From a God's eye perspective the future is already there [...] If that violates your conception of agency and free will then so be it" $(2018,313)$. 
the blockworld? But as During and Miquel remind us, above all else an expanded Bergsonisn "must engage fully with the sciences of today" — and the sciences of today are already revealing that the universe is far stranger than anyone expected a century ago. Is it inconceivable that science might reveal that the future exists?

Suppose one day time travel technology is developed, and you use it to travel to what is beyond any doubt your own future - a decade hence, or a century, or more - and then travel back to your own time to tell the tale. Such a trip would obviously provide very evidence of a very direct kind that the future is real. Faced with evidence of this strength the Bergsonian would have no option but to modify their position on the existence of the future, while retaining their other Bergsonian beliefs. Since a time machine has yet to be invented - and the development of one isn't on the horizon - we aren't likely to get direct empirical confirmation of this sort that the future exists. But evidence of an indirect kind could easily emerge. If the fundamental laws of nature take the general form we have been envisaging latterly - global constraints on four-dimensional patterns of events, with initial and final states on an equal footing - then the reality of the future would be far harder to deny. The evidence for its existence would be less direct than in the time travel case, but nonetheless potent. ${ }^{5}$ It might also pose a more serious challenge to orthodox Bergsonians than Einstein's special theory of relativity. Einstein's relativisation of simultaneity threatens our everyday understanding of the present, but it does not in itself provide powerful evidence that the entirety of the future is real. ${ }^{6}$

If we set the issue of the reality (or otherwise) of the future to one side, in other respects the stance SSM defend is altogether Bergsonian. Bergson characterises human consciousness in terms of durée, episodes of experience possessing inherent flow or dynamism. For Bergson this intrinsic flow is a basic and irreducible feature of our experience: he was adamant that our

5. SSM are by no means alone in advocating this path forward: a number of recent philosophers and physicists have argued that many of the more puzzling features of quantum theory disappear if we allow retrocausality - causal influences from the future affecting the past. This sort of approach obviously presupposes the reality of the future. For a very useful review of this work and its motivations see Friederich and Evans (2019). 6. Various ways of accomodating dynamic conceptions of time with the relativisation of simultaneity have been proposed — see Dainton (2010, ch. 19) for an introduction to some of them. One obvious tactic, for example, would be to restrict simultaneity to very small spacetime regions, and explicate temporal passage in terms of growing worldlines, rather than a universe-wide plane of simultaneity. If we move to a four-dimensional atemporal framework, this sort of move is no longer available. 
streams of consciousness do not consist of successions of instantaneous motion-free snapshots. SSM similarly take the dynamic character of consciousness to be basic and irreducible.

No less importantly, SSM's position on the relationship between consciousness and the material world is strikingly similar to Bergson's. As we saw in the previous section, SSM advocate a form of neutral monism. The $20^{\text {th }}$ century saw various attempts by analytic philosophers of mind to reduce consciousness to something else - dispositions to behave, functional properties, neural properties — but these reductive programs all proved deeply problematic, and the same applies to dualism (or so SSM maintain). Bergson did not live to see problems encountered by $20^{\text {th }}$ century reductionist programmes in the philosophy of mind, but he recognised that both dualism and reductive forms of materialism were highly problematic, and advocated a radically different approach. In the account of the physical realm that Bergson starts to elaborate in his Matter and Memory (1896) durée is not confined to human consciousness. Drawing on Maxwell's discovery of the electro-magnetic field, Bergson speculated that the physical world consists of "numberless vibrations all linked together in uninterrupted continuity, all bound up with each other and travelling in every direction like shivers running through an immense body" (Bergson 1991, 208). Although durée is to be found everywhere in the physical world, it has different "rhythms." Our consciousness is comparatively sluggish: in a second of time as measured by a clock we can experience comparatively small number of changes as (say) we watch a tennis player run to strike a ball. Over the same time interval a wave of red light undergoes a vast number of oscillations, and its consciousness consists of vast numbers of extremely brief episodes of durée. The details of Bergson's version of panpsychism are intriguing, but needn't detain us here. ${ }^{7}$ His conception of the place of consciousness in the natural world is clearly similar to that advocated by SSM.

It is not by chance that SSM gave their book Beyond the Dynamical Universe as a title. As we have seen, the view of the universe they are opposed and seeking to replace is the classical Newtonian type of physical explanation, the sort which views the world as being composed of myriad particles located in an absolute space and time, and interacting from moment-tomoment under the influences of deterministic forces. Like SSM, Bergson

7. See Čapek (1971, Part III) and Sinclair (2020, chapters 5 \& 9) for further details of Bergson's views on the nature of matter. 
rejected the absolute space and time of Newton. He was also critical of the mechanical mode of explanation. For example in Creative Evolution we find him characterising the position he was opposed to thus:

The mechanistic explanations, we said, hold good for the systems that our thought artificially detaches from the whole. But of the whole itself and of the systems which, within this whole, seem to take after it, we cannot admit a priori that they are mechanically explicable, for then time would be useless, and even unreal. The essence of mechanical explanation, in fact, is to regard the future and the past as calculable functions of the present, and thus to claim that all is given. On this hypothesis, past, present and future would be open at a glance to a superhuman intellect capable of making the calculation. Indeed, the scientists who have believed in the universality and perfect objectivity of mechanical explanations have, consciously or unconsciously, acted on a hypothesis of this kind. Laplace formulated it with the greatest precision (Bergson 1911, 37-8).

If SSM are right, and the universe runs on global variational principles, then physical systems are quite unlike the sort of mechanism Bergson is concerned with here. Their constituents are not pushed and pulled like cogs in a machine - there are, after all, no forces in the normal sense. We also need to bear in mind that our current best theory of atomic and subatomic interactions is quantum theory, and as most commonly construed, quantum mechanics is fundamentally indeterministic. Even if a scientist were equipped with complete and accurate knowledge of a particle's past, they cannot predict how events will unfold when that particle interacts with another. ${ }^{8}$ The best they can do is furnish us with various probabilities, and then wait to see what actually happens. If this picture of reality proves to be correct, then at the fundamental micro-level there are no strict dynamical laws governing how objects behave from moment to moment.

Viewing the universe as a four-dimensional ensemble governed by global laws opens up new possibilities. Emily Adlam has recently argued that even

8. To be more precise, on the orthodox Copenhagen interpretation the Schrödinger's wave equation is a fully deterministic dynamical law and indeterminism enters the picture when a measurement takes place - the wave function fixes the probability of finding a given particle at a given location. Competing fully deterministic interpretations of quantum theory do exist, such as the Many Worlds view and Bohm's hidden variables theory. On yet other approaches — in particular variants of the GRW spontaneous collapse view - Schrödinger's equation does not tell the complete story, but nature is nonetheless fundamentally indeterministic. As long as these quantum controversies remain unresolved the determinism issue remains open. But as things currently stand it is certainly very possible that the universe is fundamentally indeterministic. 
if quantum mechanics is locally indeterministic, a form of determinism may still obtain at the global level:

In a globally deterministic world, the laws of nature prescribe a unique course of history in the same way as a properly set up game of sudoku has a unique solution; the laws of nature govern the whole course of history at once, rather than moment-by-moment, in the same way as the rules of the game of sudoku apply to the whole grid at once, rather than dictating the entries column by column. Indeed, if a completed sudoku square were to be revealed column by column to an observer who could not see the whole square at once, each column would appear to be related to the previous one in a probabilistic way (there would be obvious patterns of dependence which could be described by probabilistic rules, but there would not usually be enough information available to determine the next column exactly), and I suggest that similarly, the apparently probabilistic nature of quantum theory is a consequence of our inability to see the whole picture (Adlam 2018, 43-44).

This is an intriguing picture - and the sudoku analogy is quite brilliant. A universe where Adlam's type of global determinism obtains is certainly even less Bergsonian in character than a universe which the future is real but in which the physical laws are fundamentally non-determinisic. But since at this point it is by no means clear that our universe is in fact globally deterministic — it may turn out that the laws governing the universe are global but only probabilistic - perhaps Bergsonians need not lose too much sleep over this issue just yet.

\section{Blockworld Bergsonism}

There is a further potential obstacle standing in the way of a "Blockworld Bergsonism" as we might call it. Let's assume that consciousness is irreducibly dynamic in the way both camps claim. Does it really make sense to suppose that experience with this character can exist in a universe of the block type - in a universe where everything is equally real, and where there is no genuine coming into being?

To bring matters into clearer focus, let's consider a simple stream of consciousness that consists of a succession of brief auditory tones, C-DE-F. Let's further assume that this stream consists of a sequence of partially overlapping temporally extended experiential episodes: [C-D], [D-E], $[\mathrm{E}-\mathrm{F}]$. This, of course, is assuming an extensional model of temporal experience - which I have elsewhere suggested is how successive episodes of Bergson's durée should be seen as related when they unfold in the same stream consciousness (see Dainton 2017). 

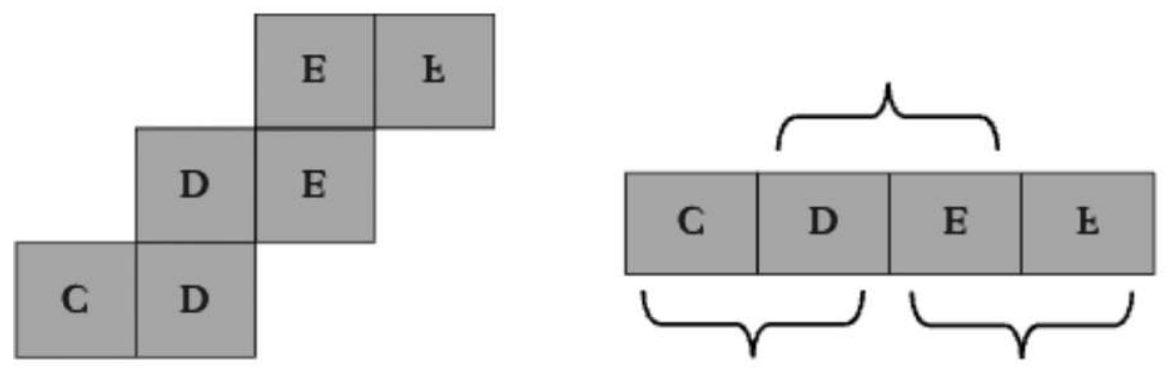

FIGURE 2. Two ways in which temporal experience could exist in time

The figure on the left depicts how a presentist might construe our simple stream of consciousness. ${ }^{9}$ An initial episode of durée C-D gives way to a (partially) new episode D-E; this state of affairs comes about by virtue of tone C's ceasing to exist and tone E coming into existence - the D-tone is depicted twice over, but this is simply for graphical clarity, it is not experienced twice-over. When $\mathrm{D}$ in turn ceases to exist and $\mathrm{F}$ comes into existence we have another (partially) new episode of durée E-F. In sharp contrast the figure on the right is a depiction of how an eternalist or block theorist views matters. All four tones are fully and equally real; the brackets represent the fact that $\mathrm{C}-\mathrm{D}$ are experienced together in a single episode of durée, as are $\mathrm{D}-\mathrm{E}$ and $\mathrm{E}-\mathrm{F}$.

The question we need to address is straightforward: does the block theorist's construal on the right really make sense, metaphysically and phenomenologically? Or do we need something akin to the state of affairs depicted on the left? As far as I can see, from a phenomenological standpoint the two models are perfectly equivalent. In both cases tone $\mathrm{C}$ is experienced as being followed by tone D, D is experienced as being followed by E, and $\mathrm{E}$ is experienced as being followed by F. Focusing on [D-E], it seems inevitable that that the phenomenal character of this experiential episode will be precisely the same in both cases. The only difference between the two scenarios is that for the presentist the experiences of $\mathrm{C}$ and $\mathrm{F}$ do not exist at all during the period when $[\mathrm{D}-\mathrm{E}]$ is being experienced, whereas for

9. Since an experience C-D extends a brief way through time, so too does the present. More orthodox versions of presentism which hold that the present is instantaneous are not compatible with the extensional view of temporal experience. For further discussion of this "extended" or "compound" mode of presentism see Dainton (2010, chapters 6 and 7). 
the eternalist or block theorist they do. But how can the existence or nonexistence of $\mathrm{C}$ and $\mathrm{F}$ in the past or future affect the phenomenal character of the experiences in [D-E]? The character of this experience will be fully determined by the intrinsic properties of $\mathrm{D}$ and $\mathrm{E}$, and these are precisely the same in the two cases. It is of course the case that as the succession [DE] unfolds this auditory perceptual experience is accompanied by acoustic memories of the recently experienced C-tone. But this sort of memory is equally at home in both presentist and block models.

I do not want to suggest this is the last word on this issue. Perhaps there is a way in which the coming-into-existence of an experience can create phenomenal effects that simply cannot exist in a blockworld. But if so this is a good deal less obvious than one might have supposed. As a consequence it may well be that the very existence of Bergsonian durée does not require the unreality of the future - and if this should prove to be the case it would have important implications for the possible forms an expanded Bergsonism might take. ${ }^{10}$

\section{Bibliography}

Adlam, Emily. 2018. "Quantum Mechanics and Global Determinism." Quanta 7(1): 40-53.

Bergson, Henri. 1911. Creative Evolution. Translated by Arthur Mitchell. New York: Henry Hold and Company.

Bergson, Henri. 1965. Duration and Simultaneity. Translated by Leon Jacobson. New York: Bobbs-Merril Company.

Bergson, Henri. 1991. Matter and Memory. Translated by Nancy Margaret Paul and W. Scott Palmer. New York: Zone Books.

Čapek, Milič. 1971. Bergson and Modern Physics. Dordrecht: Reidel.

Chiang, Ted. 2002. Starlight 2. New York: Tor.

Dainton, Barry. 2008. The Phenomenal Self. Oxford: Oxford University Press.

Dainton, Barry. 2010. Time and Space. London: Routledge.

10. My thanks to two anonymous referees for helpful comments. An earlier version of this article appeared (in Japanese) in 2018. Rebooting Bergson's Matter and Memory: Multidisciplinary Perspectives on Expanded Bergsonism edited by Yasushi Hirai, Hisashi Fujita, Shin Abiko, 189-223. Tokyo: Shoshi Shinsui. I am grateful for permission to reproduce this material here. 
Dainton, Barry. 2017. "Bergson on Temporal Experience and Durée Reélle." In The Routledge Handbook of Temporal Experience edited by Ian Phillips, 93-106. Abingdon: Routledge, 2017.

During, Élie, and Paul-Antoine Miquel. 2020. "We Bergsonians: The Kyoto Manifesto." Parrhesia 33: 17-42.

Feynman, Richard, Robert Leighton, and Matthew Sands. 2011. The Feynman Lectures on Physics Volume II. New York: Basic Books.

Friederich, Simon and Peter W Evans. 2019. "Retrocausation in Quantum Mechanics.” Stanford Encyclopedia of Philosophy. https://plato.stanford. edu/entries/qm-retrocausality/.

Le Poidevin, Robin. 2013. "Time and Freedom." In A Companion to the Philosophy of Time edited by Adrian Bardon and Heather Dyke, 535548. New York: John Wiley and Sons.

Maupertuis, Pierre Louis (De). 1744. "Accord des Différent Lois de la Nature qui Avaient, Jusqu’à ici, Paru Incompatible.” Mémoires de l'Academie des Sciences de Paris.

Silberstein, Michael, W. Mark Stuckey and Timothy McDevitt. 2018. Beyond the Dynamical Universe: Unifying Block Universe Physics and Time as Experienced. Oxford: Oxford University Press.

Sinclair, Mark. 2020. Bergson. London: Routledge.

Strawson, Galen. 2006. "Panpsychism?" In Consciousness and its Place in Nature edited by Anthony Freeman, 184-208. Exeter: Imprint Academic.

Villeneuve, Denis, 2016. Arrival. 21 Laps Entertainment.

Wharton, Ken. 2015. “The Universe is not a Computer." In Questioning the Foundations of Physics: Which of Our Fundamental Assumptions Are Wrong? edited by Anthony Aguirre, Brendan Foster and Zeeya Merali, 177-189. New York: Springer.

Wolchover, Natalie. 2013. "A Jewel at the Heart of Physics." Quanta https:// www.quantamagazine.org/physicists-discover-geometry-underlyingparticle-physics-20130917/. 
Barry Dainton's interests are mainly in the philosophy of mind and metaphysics. He spends a lot of time thinking about the nature of consciousness and its relationship with the rest of the universe. He is also interested in the nature of the self, the various metaphysical implications associated with time and space, and the philosophical implications of advances in science and technology. Thus far he has published: Stream of Consciousness (Routledge, 2000), Time and Space (Acumen 2001,2nd edition Routledge 2010), The Phenomenal Self (Oxford University Press, 2008), and Self (Penguin, 2014). He has also coedited The Bloomsbury Companion to Analytic Philosophy (Bloomsbury, 2014), and Minding the Future: Artificial Intelligence, Philosophical Visions and Science Fiction (Springer, 2021).

\begin{abstract}
Given Bergson's commitment to a dynamic conception of time and an open future it seems likely that he would not have welcomed developments in physics which suggest that we live in an eternal four-dimensional block-universe. The route from Einstein's special theory of relativity to the eternalist conception of time has been thoroughly explored - and Bergson himself explored ways of reconciling his own doctrines with Einstein's theory. But there is more than one route from physics to the block-universe. The route I am concerned with here may be less familiar than Einstein's, but is potentially of considerable significance since some contemporary physicists believe it may well be the physics of the future. Denis Villeneuve's recent movie Arrival together with the science fiction story by Ted Chiang it is based upon - provide useful points of entry into the relevant science.
\end{abstract}

Keywords: expanded bergsonism, blockworld, variational principles, Arrival (film), adynamical explanation.

Résumé : Étant donné l'engagement de Bergson pour une conception dynamique du temps et un avenir ouvert, il semble probable qu'il n'aurait pas accueilli favorablement les développements de la physique qui suggèrent que nous vivons dans un éternel univers de blocs à quatre dimensions. Au cours des dernières décennies, la route menant de la théorie de la relativité restreinte d'Einstein à la conception éternelle du temps a été explorée en profondeur - et Bergson lui-même a exploré les moyens de concilier ses propres doctrines avec la théorie d'Einstein. Mais il y a plus d'une voie de la physique vers le blocunivers. La route qui m'intéresse ici est peut-être moins familière que celle d'Einstein, mais peut revêtir une importance considérable, car certains physiciens contemporains pensent qu'il pourrait bien s'agir de la physique de l'avenir. Le récent film "Arrival" de Denis Villeneuve, ainsi que l'histoire de science-fiction de Ted Chiang sur laquelle il est basé, fournissent des points d'entrée utiles dans ces questions.

Mots-clés : bergsonisme étendu, blockworld, principes variationnels, Arrival (film), explication adynamique. 
